\title{
Propagation and Relaxation of Tension in Stiff Polymers
}

\author{
Oskar Hallatschek, ${ }^{1}$ Erwin Frey, ${ }^{1,2}$ and Klaus Kroy ${ }^{1,3}$ \\ ${ }^{1}$ Hahn-Meitner Institut, Glienicker Straße 100, 14109 Berlin, Germany \\ ${ }^{2}$ Arnold Sommerfeld Center, Department of Physics, Ludwig-Maximilians-Universität München, \\ Theresienstraße 37, 80333 München, Germany \\ ${ }^{3}$ Institut für Theoretische Physik, Universität Leipzig, Augustusplatz 10/11, 04109 Leipzig, Germany
}

(Received 18 May 2004; published 25 February 2005)

\begin{abstract}
We present a unified theory for the longitudinal dynamic response of a stiff polymer in solution to various external perturbations (mechanical excitations, hydrodynamic flows, electrical fields, temperature quenches, etc.) that can be represented as sudden changes of ambient/boundary conditions. The theory relies on a comprehensive analysis of the nonequilibrium propagation and relaxation of backbone stresses in a wormlike chain. We recover and substantially extend previous results based on heuristic arguments. New experimental implications are pointed out.
\end{abstract}

Despite considerable practical and interdisciplinary interest, it is theoretically not yet fully understood how polymers respond to external fields [1,2]. Consider, e.g., the simple problem of an inextensible semiflexible polymer suddenly stretched along its end-to-end vector by an external force $\mathfrak{f}$ (pulling). It was recognized before [3] that tension propagation (from the ends into the bulk) is the key to understanding its dynamics: in response to the spreading tension, the polymer stretches within a growing boundary layer of length $\ell_{\|}$, as depicted in Fig. 1. Depending on the setup, different tension propagation laws $\ell_{\|}(t)$ have been predicted [3-6]. In particular, we contrast the above pulling scenario with the (inverse) release scenario, where a constant prestretching force $\mathfrak{f}$ is suddenly removed. While for small $\mathfrak{f}$ one expects $\ell_{\|}(t) \propto t^{1 / 8}$ in both cases [4], the predictions for strong force are markedly different: $\ell_{\|}(t) \propto$ $(\mathfrak{f} t)^{1 / 4}$ [3] for pulling and $\ell_{\|}(t) \propto \mathfrak{f}^{3 / 4} t^{1 / 2}$ [5] for release. However, the precise meaning of "strong" and "weak," and the validity of the diverse assumptions in Refs. [3,5] are not obvious. Here we develop from first principles a theory of stress propagation and relaxation that allows us to unify and systematically extend these previous results and to derive (often analytically) the longitudinal nonlinear response to various external perturbations. After introducing the standard model of a semiflexible polymer, we extend a heuristic argument of Ref. [4] for pulling. This elucidates the crossover from "weak-force" to "strongforce" behavior and reveals the crucial length-scale separation underlying our subsequent systematic analysis.

The wormlike chain model represents the polymer by a smooth inextensible contour $\mathbf{r}(s, t)$ subject to the energy

$$
\mathcal{H}=\frac{\kappa}{2} \int_{0}^{L} d s \mathbf{r}^{\prime \prime 2}+\frac{1}{2} \int_{0}^{L} d s f \mathbf{r}^{\prime 2} .
$$

The scalar force $f(s, t)$ (backbone "tension") is a Lagrange multiplier function introduced [7] to enforce the inextensibility constraint $\mathbf{r}^{\prime 2}=1$ for the tangent vector $\mathbf{r}^{\prime} \equiv$ $\partial \mathbf{r} / \partial s$. We require a bending stiffness $\boldsymbol{\kappa}$ such that the persistence length $\ell_{p}=\kappa / k_{B} T$ is much larger than the contour length $L$, which entails relative mean square transverse displacements of order $\epsilon \equiv L / \ell_{p} \ll 1$. The elastic forces derived from $\mathcal{H}$ have to balance thermal forces $\boldsymbol{\xi}$ (Gaussian white noise) and Stokes friction, which (in the "free-draining" approximation) enters through two local friction coefficients per unit length $\zeta_{\perp}, \zeta_{\|} \approx \zeta_{\perp} / 2$ for motion perpendicular and parallel to $\mathbf{r}^{\prime}$, respectively: $\left[\zeta_{\|} \mathbf{r}^{\prime} \mathbf{r}^{\prime}+\zeta_{\perp}\left(1-\mathbf{r}^{\prime} \mathbf{r}^{\prime}\right)\right] \cdot \partial_{t} \mathbf{r}=-\delta \mathcal{H} / \delta \mathbf{r}+\xi$. For the following, we choose convenient units such that $\kappa \equiv \zeta_{\perp} \equiv 1$. Then, all dimensional quantities represent powers of length (e.g., $k_{B} T=\ell_{p}^{-1}$ ) and $\zeta_{\|} \equiv \zeta \approx 1 / 2$.

We now turn to a heuristic discussion of pulling to leading order in $\epsilon$. In the parametrization introduced in Fig. 1, the exact equations of motion reduce to an equation for the transverse displacements $\mathbf{r}_{\perp}$ alone,

$$
\partial_{t} \mathbf{r}_{\perp}=-\mathbf{r}_{\perp}^{\prime \prime \prime \prime}+f \mathbf{r}_{\perp}^{\prime \prime}+\boldsymbol{\xi}_{\perp},
$$

with a spatially uniform tension $f=\mathfrak{f} \Theta(t)$ fixed by the driving force at the boundaries; $\left\langle\boldsymbol{\xi}_{\perp}(s, t) \cdot \boldsymbol{\xi}_{\perp}\left(s^{\prime}, t^{\prime}\right)\right\rangle=$ $4 \ell_{p}^{-1} \delta\left(s-s^{\prime}\right) \delta\left(t-t^{\prime}\right)$. The (higher order) longitudinal displacements $r_{\|}$are "slaved" by the arclength constraint $r_{\|}^{\prime}=\mathbf{r}_{\perp}^{\prime 2} / 2$.

From a simple scaling analysis of Eq. (2), $\left\langle\mathbf{r}_{\perp}\right\rangle / t \approx$ $\left\langle\mathbf{r}_{\perp}\right\rangle\left(\ell_{\perp}^{-4}+\mathfrak{f} \ell_{\perp}^{-2}\right)$, we deduce the characteristic dynamic wavelength $\ell_{\perp}(t)$ corresponding to the (lowest) mode equilibrated at time $t$. For instance, $\ell_{\perp}\left(t_{L}^{\perp}\right) \equiv L$ defines the longest relaxation time. Because of the competition between bending forces $\left(\propto r_{\perp} \ell_{\perp}^{-4}\right)$ and tension $\left(\propto r_{\perp} \mathfrak{f} \ell_{\perp}^{-2}\right)$, the growth of $\ell_{\perp}$ exhibits a dynamic crossover from free

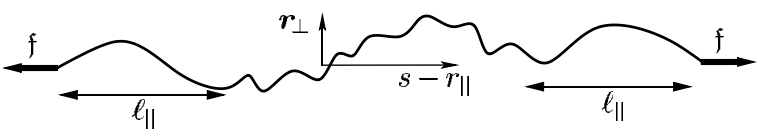

FIG. 1. Pulling (schematic): In response to an external force $\mathfrak{f}$, the thermally undulated contour $\mathbf{r}(s)=\left(\mathbf{r}_{\perp}, s-r_{\|}\right)^{T}$ is straightened within boundary layers of growing width $\ell_{\|}(t)$. 
TABLE I. The transverse equilibration length $\ell_{\perp}(t)$ and the tension propagation length $\ell_{\|}(t)$ both exhibit a crossover at $t_{\uparrow} \equiv$ $\mathfrak{f}^{-2}$ (here, for the pulling problem with $\mathfrak{f} \gg L^{-2}, t \ll t_{L}^{\perp}$ ).

\begin{tabular}{lcc}
\hline \hline & $\ell_{\perp}(t)$ & $\ell_{\|}(t)$ \\
\hline$t \ll t_{\mathfrak{f}}$ & $t^{1 / 4}$ & $t^{1 / 8}\left(\ell_{p} / \zeta\right)^{1 / 2}[4]$ \\
$t \gg t_{\mathfrak{f}}$ & $t^{1 / 2} \mathfrak{\uparrow}^{1 / 2}$ & $t^{1 / 4} \mathfrak{f}^{1 / 4}\left(\ell_{p} / \zeta\right)^{1 / 2}[3]$ \\
\hline \hline
\end{tabular}

relaxation $\left(\ell_{\perp} \propto t^{1 / 4}\right)$ to relaxation under tension $\left(\ell_{\perp} \propto\right.$ $\sqrt{\mathfrak{f} t}$ ) at a characteristic time $t_{\mathfrak{f}} \equiv \mathfrak{f}^{-2}$ (Table I, left-hand side).

By the above interpretation of $\ell_{\perp}$, the longitudinal elongation of a subsection of arclength $\ell_{\perp}$ under a given tension $\mathfrak{f}$ can be estimated by equilibrium theory. One has to distinguish weak and strong tension relative to the internal characteristic force scale $\ell_{\perp}^{-2}$ of the subsection, which corresponds to the Euler buckling force of the subsection. For weak tension $\mathfrak{\uparrow} \ll \ell_{\perp}^{-2}$, the elongation $\mathfrak{\uparrow} \ell_{\perp}^{4} \ell_{p}^{-1}$ follows from linear response [8]. For strong tension $\mathfrak{f} \gg$ $\ell_{\perp}^{-2}$ the subsection is virtually straight, so that the elongation is equal to its equilibrium thermal contraction $\ell_{\perp}^{2} \ell_{p}^{-1}$ caused by the bending undulations. Since the whole polymer is subject to the same constant tension $\mathfrak{f}$, it can be divided (at any time $t$ ) into $L / \ell_{\perp}(t)$ independent equilibrated subsections of length $\ell_{\perp}(t)$. The total change $\Delta R(t) \equiv|\langle R(t)-R(0)\rangle|$ of the end-to-end distance $R$ thus crosses over from $\Delta R \propto L \mathfrak{\uparrow} \ell_{\perp}^{3} \ell_{p}^{-1} \propto t^{3 / 4}$ [9] for $t \ll$ $t_{\mathfrak{f}}$ to $\Delta R \propto L \ell_{\perp} \ell_{p}^{-1} \propto t^{1 / 2}$ for $t \gg t_{\mathfrak{f}}$.

These results comprise the predictions of ordinary perturbation theory (OPT) to leading order. As evident from Eq. (2), it neglects longitudinal friction forces, which are of higher order in $\epsilon$. However, the resulting $\Delta R$ obtained above implies a total longitudinal friction $\zeta L \partial_{t} \Delta R$ crossing over from $\zeta L^{2} \tilde{\mathfrak{f}} t^{-1 / 4} \ell_{p}^{-1}$ to $\zeta L^{2}(\tilde{\mathfrak{f}} / t)^{1 / 2} \ell_{p}^{-1}$ at $t_{\mathfrak{f}}$. Both expressions diverge $[4,10]$ for $t \rightarrow 0$ indicating the breakdown of OPT at short times. More precisely, for times shorter than a certain $t_{\star}$ [11] the longitudinal friction would exceed the driving force $\mathfrak{f}$. This was recognized as a consequence of the omission of tension propagation in Eq. (2): it was argued [4] that actually only segments up to a distance $\ell_{\|}(t)$ [12] from the ends are set into longitudinal motion causing longitudinal friction. The proper expression for the total longitudinal friction thus follows from the above upon substituting $L$ by $\ell_{\|}$. The balance of longitudinal friction and external force can now be satisfied by choosing the size $\ell_{\|}(t)$ of the boundary layer according to Table I (right-hand side). Hence, the putative weak-force and strong-force cases $\ell_{\|} \propto t^{1 / 8}[4]$ and $\ell_{\|} \propto t^{1 / 4}$ [3] are identified as asymptotes of a "short-long time" crossover (still) signaling the change from "free" to "forced" relaxation at $t=t_{\mathrm{f}}$.

In summary, the foregoing discussion reveals (i) the breakdown of OPT at times $t<t_{\star}$, where (ii) the neglected longitudinal friction actually limits the relaxation to boundary layers of size $\ell_{\|}$, (iii) the crossover from free to forced relaxation at $t=t_{\mathfrak{f}}$, and (iv) the scale separation $\ell_{\perp} / \ell_{\|} \propto \epsilon^{1 / 2} \ll 1$ (Table I).

The latter lends itself as starting point for a multiplescale calculus to separate the physics on different dynamic scales and obtain an improved (multiple-scale) perturbation theory (MSPT) that is regular in the limit $t \rightarrow 0$ while $\epsilon \ll 1$ is fixed. The procedure is similar to that for athermal dynamics [13] and will be documented in detail elsewhere [14]. The basic idea is to regard functions $g(s)$ as functions $g\left(s, \bar{s} \epsilon^{1 / 2}\right)$ of rapidly and slowly varying arclength parameters $s$ and $\bar{s} \epsilon^{1 / 2}$ that are treated as independent variables. Closed equations for the dynamics on the scale $\bar{s} \epsilon^{1 / 2}$ are obtained upon averaging $\bar{g}(\bar{s}) \equiv$ $\int_{l} d s g\left(s, \bar{s} \epsilon^{1 / 2}\right) / l$ over the fluctuations on the microscale $\left(\ell_{\perp} \ll l \ll \ell_{\|}\right)$. To leading order, we get [14] $f=\bar{f}(\bar{s}, t)$,

$$
\partial_{t} \mathbf{r}_{\perp}=-\mathbf{r}_{\perp}^{\prime \prime \prime \prime}+\bar{f} \mathbf{r}_{\perp}^{\prime \prime}+\xi_{\perp}, \quad \partial_{\bar{s}}^{2} \bar{f}=-\zeta \overline{\partial_{t} r_{\|}^{\prime}} .
$$

This provides the sought-after rigorous local generalization of the above, heuristically renormalized force balance. The arclength average extending over many $\left(l / \ell_{\perp} \gg 1\right)$ uncorrelated sections of length $\ell_{\perp}$ subject to the same tension $\bar{f}(\bar{s})$ can be interpreted as a coarse graining that effectively generates a local ensemble average: $\overline{\partial_{t} r_{\|}^{\prime}} \sim$ $\left\langle\partial_{t} r_{\|}^{\prime}\right\rangle$ for $\epsilon \rightarrow 0$. Only the "systematic" $\mathcal{O}(1)$ variations of the tension are retained, while its $\mathcal{O}(\boldsymbol{\epsilon})$ noise is leveled out, so that the longitudinal Eq. (3) represents deterministic dynamics: local longitudinal motion is driven by tension gradients (like in a thread pulled through a viscous medium).

Integrating the longitudinal Eq. (3) over time expresses the change of the thermal contraction $r_{\|}$in terms of the time-integrated tension $F \equiv \int_{0}^{t} d t^{\prime} f$, namely, $\left\langle r_{\|}^{\prime}(\bar{s}, t)-\right.$ $\left.r_{\|}^{\prime}(\bar{s}, 0)\right\rangle=-\partial_{\bar{s}}^{2} \bar{F} / \zeta$. Since $2 r_{\|}^{\prime}=\mathbf{r}_{\perp}^{\prime 2}$ from the arclength constraint, we integrate the transverse Eq. (3) for $\left\langle\mathbf{r}_{\perp}^{\prime 2}\right\rangle \times$ $(\bar{s}, t)=\left\langle\left[\frac{1}{L} \sum_{q} \int_{-\infty}^{t} d t^{\prime} q \chi_{\perp}\left(q ; t, t^{\prime}\right) \xi_{\perp}\left(q, t^{\prime}\right)\right]^{2}\right\rangle$. Here

$$
\chi_{\perp}\left(q ; t, t^{\prime}\right) \equiv e^{-q^{4}\left(t-t^{\prime}\right)-q^{2}\left[\bar{F}(\bar{s}, t)-\bar{F}\left(\bar{s}, t^{\prime}\right)\right]}
$$

should be recognized as the susceptibility for the response of the Fourier modes of $\mathbf{r}_{\perp}$ to transverse forces. Note that the $\bar{s}$ dependence of $\left\langle\mathbf{r}_{\perp}^{/ 2}\right\rangle$ is purely adiabatic, as it is parametrically inherited from $\bar{F}(\bar{s}, t)$. Altogether, Eq. (3) is condensed into a single equation for $\bar{F}$ [15],

$$
\frac{\partial_{\bar{s}}^{2} \bar{F}}{\zeta}=\int_{0}^{\infty} \frac{d q}{\pi}\left[\frac{1-\chi_{\perp}^{2}(q ; t, 0)}{\ell_{p}^{-}\left(q^{2}+f^{-}\right)}-\frac{2 q^{2}}{\ell_{p}} \int_{0}^{t} d t^{\prime} \chi_{\perp}^{2}\left(q ; t, t^{\prime}\right)\right] .
$$

Indices "- " referring to $t<0$ were introduced to allow the system to be prepared in equilibrium with ambient/ boundary conditions different from those prescribed for $t \geq 0$. Taking $\ell_{p}$ (for $t \geq 0$ ) to $\infty$, only the first term in the integrand remains, which thus accounts for the deterministic relaxation of the initial thermal contraction (set by $\ell_{p}^{-}$, $\left.f^{-}\right)$. In this limit, the "zero-temperature" buckling dynamics analyzed in Ref. [13] is recovered. For finite $\ell_{p}$ the 
second term in the brackets represents the action of the thermal forces for $t>0$, which aim to establish the equilibrium contraction.

To further unravel the physical implications of Eq. (5), we begin with the tension propagation regime $\ell_{\|} \ll L$, where $L$ is irrelevant. Problems like pulling and release still depend on four length scales $\left(\ell_{p}^{-}=\ell_{p}, \mathfrak{f}^{-1 / 2}, s, t^{1 / 4}\right)$. Yet, Eq. (5) is solved exactly by the two-variable scaling ansatz

$$
f(\bar{s}, t)=\mathfrak{f} \Phi\left(\bar{s} / s_{\mathfrak{f}}, t / t_{\mathfrak{f}}\right) .
$$

With $t_{\mathfrak{f}} \equiv \mathfrak{f}^{-2}$ and $s_{\mathfrak{f}} \equiv\left(\ell_{p} / \zeta\right)^{1 / 2} t_{\mathfrak{f}}^{1 / 8}$, Eq. (6) can be shown to eliminate the parameter dependencies in Eq. (5) and in its boundary conditions. The scaling function $\Phi$ describes how sudden changes of the tension at the ends spread into the bulk of the polymer. In the limits $t \ll t_{\mathfrak{f}}$ and $t \gg t_{\mathrm{f}}$ Eq. (6) reduces to the simple (one-variable) scaling laws $\Phi \sim\left(t / t_{\mathfrak{F}}\right)^{\alpha} \phi\left(\bar{s} / \ell_{\|}\right)$with the tension propagation length $\ell_{\|} \equiv s_{\mathfrak{f}}\left(t / t_{\mathfrak{f}}\right)^{z}$. Note that this crossover scenario, as well as the expressions for $t_{\mathfrak{f}}, s_{\mathfrak{f}} \approx \ell_{\|}\left(t_{\mathfrak{f}}\right)$, and $\ell_{\|}$are consistent with our above heuristic observations for pulling (Table I). In fact, this structure generally emerges for all problems involving a single external force scale. It is implicitly understood that $\phi, \alpha$, and $z$ will generally not only depend on the kind of external perturbation applied, but will also be different in both scaling limits $t / t_{\mathfrak{f}} \rightarrow 0, \infty$. In the following, these limits are analyzed in more detail.

For $t \ll t_{\mathfrak{f}}$ Eq. (5) may be linearized in $f$ and the scaling function $\phi$ can be obtained analytically [14]. In contrast to $\phi$, the corresponding exponent $z=1 / 8$ does not depend on the boundary conditions. It already follows from requiring $\phi$ to become $\mathfrak{f}$ independent, as in linear response. The short-time dynamics for strong external force is thus closely related to the linear response. As established by our heuristic discussion of pulling, this is due to the relaxation of subsections with Euler forces $\ell_{\perp}^{-2}$ much larger than the external force. Note, however, that the limit $\mathfrak{f} \rightarrow 0$ is problematic, as it does not interchange with $\epsilon \rightarrow 0$ [16]. In fact, extending Eq. (5) to linear response amounts to an uncontrolled factorization approximation $\left\langle f r_{\perp}^{2}\right\rangle \rightarrow\langle f\rangle\left\langle r_{\perp}^{2}\right\rangle$.

For $t \gg t_{\mathfrak{f}}$ the dynamics becomes nonlinear in the external force and starts to depend on the force protocol. Previously predicted power laws can be recovered from Eq. (5) by employing different approximations to its righthand side. In the taut-string approximation of Ref. [3] one neglects for $t>0$ bending and thermal forces against the tension; i.e., one drops the $q^{4}$ term in the expression Eq. (4) for $\chi_{\perp}$ and sets $\ell_{p} \rightarrow \infty$. The complementary quasistatic approximation of Ref. [5] amounts to the omission of memory effects, i.e., to the assumption of instantaneous equilibration of tension and stored length, $F(t)-F\left(t^{\prime}\right) \rightarrow$ $f(t)\left(t-t^{\prime}\right)$. A careful analysis of Eq. (5) [14] shows that either of these approximations becomes rigorous in the intermediate asymptotic regime defined by $t \gg t_{f}, \ell_{\|} \ll L$.

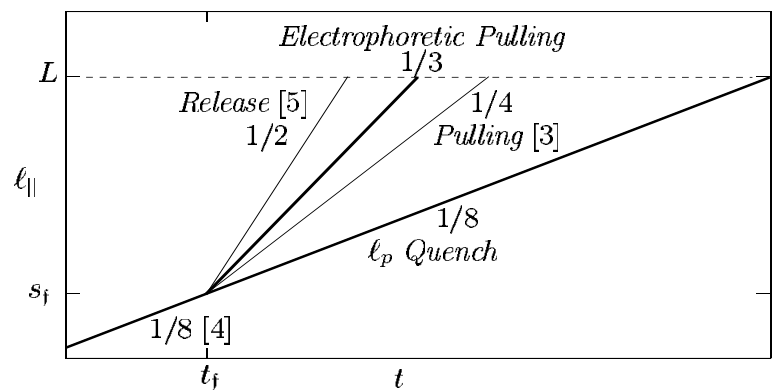

FIG. 2. Double-logarithmic sketch of the tension propagation laws $\ell_{\|}(t) \propto t^{z}$. At $t_{\mathfrak{f}}=\mathfrak{f}^{-2}$ they cross over from a universal short-time regime to (problem-specific) tension-dominated intermediate asymptotics, except for weak forces, $\mathfrak{\uparrow}<\ell_{p}^{2} / L^{4}$, and for $\ell_{p}$ quenches. The propagation ends when $\ell_{\|}(t) \approx L$.

The "pure" [17] scenarios are summarized in Fig. 2 and for the cases pulling and release also in Fig. 3 and Table II.

In Fig. 2 we have, moreover, displayed results for sudden changes in persistence length from $\ell_{p}^{-}$to $\ell_{p} \neq \ell_{p}^{-}\left(\ell_{p}\right.$ quench) and electrophoretic pulling, which have not been discussed before. The second is a variant of the pulling problem, where the external force is applied along the whole contour of an end-grafted polymer as is the case for a hydrodynamic flow or an electric field. The $\ell_{p}$ quench is exceptional in that there is no external force scale involved, so that Eq. (5) can be solved by a simple onevariable scaling ansatz $f(\bar{s}, t)=t^{-1 / 2} \varphi\left(\bar{s} / \ell_{\|}\right)$with $\ell_{\|} \approx$ $\left(\ell_{p} / \zeta\right)^{1 / 2} t^{1 / 8}$. Neither the taut-string approximation nor the quasistatic approximation can be applied, and in contrast to the other cases the scaling function has to be evaluated numerically.

Eventually, at a time $t_{L}^{\|}$, the tension will have propagated through the whole polymer, i.e., $\ell_{\|}\left(t_{L}^{\|}\right) \approx L$. Subsequently, the characteristic longitudinal scale is the contour length $L$.

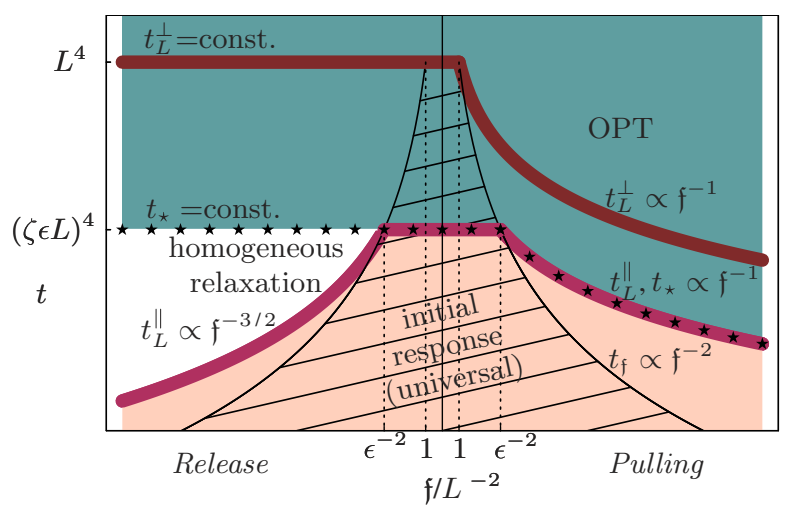

FIG. 3 (color online). Characteristic times (logarithmic scale) for pulling and release against the applied external force $\mathfrak{f}$ (linear scale). The time $t_{\star}$ (stars) separates regions where ordinary perturbation theory (OPT) applies (dark shaded) from regions (light shaded) of linear (hatched) and nonlinear tension propagation and from homogeneous tension relaxation (white). Whereas longitudinal friction is negligible for $t>t_{\star}$, it limits the dynamics for $t<t_{\star}$. 
TABLE II. Growth laws for the end-to-end distance $\Delta \bar{R}(t)$ in the intermediate asymptotic regimes marked in Fig. 3. OPT and MSPT refer to "ordinary perturbation theory" and "multiple-scale perturbation theory," respectively.

\begin{tabular}{|c|c|c|}
\hline & Release & Pulling \\
\hline Linear MSPT & \multicolumn{2}{|c|}{$\begin{array}{c}2^{5 / 8} \Gamma\left(\frac{15}{8}\right)^{-1}\left(\zeta \ell_{p}\right)^{-1 / 2} \mathfrak{f} t^{7 / 8} \\
2^{-3 / 4} \Gamma\left(\frac{7}{7}\right)^{-1}\left(L / \ell_{p} \mathfrak{f} t^{3 / 4}\right.\end{array}$} \\
\hline Linear OPT [9] & & \\
\hline Nonlinear MSPT & $3.503\left(\zeta \ell_{p}\right)^{-1 / 2} \mathfrak{f}^{1 / 4} t^{1 / 2}$ & $\left(\frac{512}{9 \pi}\right)^{1 / 4}\left(\zeta \ell_{p}\right)^{-1 / 2}(\mathfrak{f} t)^{3 / 4}$ \\
\hline Homogeneous relaxation & $2 \times 54^{2 / 3}\left(L / \zeta \ell_{p}^{2}\right)^{1 / 3} t^{1 / 3}$ & - \\
\hline Nonlinear OPT & $2^{3 / 4} \Gamma\left(\frac{1}{4}\right)^{-1}\left(L / \ell_{p}\right) t^{1 / 4}$ & $(2 / \pi)^{1 / 2}\left(L / \ell_{p}\right)(\mathfrak{f} t)^{1 / 2}$ \\
\hline
\end{tabular}

One would expect that regular perturbation theory would then become valid. Surprisingly, for $t_{L}^{\|} \gg t_{f}$ the release scenario provides an exception. The contraction dynamics exhibits an intermediate regime of homogeneous tension relaxation (white in Fig. 3). Its asymptotic power-law form is revealed by the same quasistatic approximation applicable during the preceding tension propagation, but with the separation ansatz $f(\bar{s}, t) \sim h(\bar{s})\left(\zeta L^{2} / \ell_{p} t\right)^{2 / 3}$ instead of the single-variable scaling ansatz. It solves Eq. (5) analytically with a roughly parabolic stationary tension profile $h(\bar{s})$. The homogeneous tension relaxation dominates the short-time relaxation for $t \ll t_{\star}=\left(\zeta L^{2} / \ell_{p}\right)^{4}$ if $\mathfrak{\uparrow} \rightarrow \infty$ (i.e., $t_{L}^{\|} \rightarrow 0$ ).

To make contact with experiments, it is desirable to derive the consequences for pertinent observables such as the end-to-end distance. Integrating the longitudinal Eq. (3) over $\bar{s}$ and $t$ shows that the growth laws $\Delta \bar{R}(t)=$ $2 \zeta^{-1}\left|\bar{F}^{\prime}(\bar{s}=0, t)\right|$ are directly related to the tension profiles discussed above. Table II summarizes our results for the intermediate asymptotic regimes. Note that $\Delta \bar{R}$ is a coarse-grained quantity that does not resolve the "microscopic" details below the coarse-graining scale $l$. Near the polymer ends these give relevant contributions obliterating the predicted $t^{7 / 8}$ in experiments [4,18]. During homogeneous tension relaxation $\Delta \bar{R} \propto t^{1 / 3}$, which we expect to hold for strongly stretched polymers even if $L \gg \ell_{p}$ (e.g., DNA), at variance with earlier predictions [5]. The exponent $1 / 3$ coincides with that obtained by adiabatic application of the stationary force-extension relation [19] to a "frictionless" [2] polymer with attached beads at its ends. Finally, $\Delta \bar{R} \approx\left(\zeta \ell_{p}\right)^{-1 / 2} t^{3 / 8}$ in $\ell_{p}$ quenches for $t \ll t_{L}^{\|}$. Interestingly, the tension propagation/relaxation itself can in some situations be directly monitored experimentally. In $\ell_{p}$ quenches, the (longitudinal) radius of gyration mirrors the characteristic bulk relaxation $f \propto t^{-1 / 2}$ of the tension [13]. In electrophoretic pulling, where $\Delta \bar{R} \propto t$ for $t \ll t_{L}^{\|}$, the force on the grafted end obeys $\mathfrak{f} \propto \ell_{\|}$.

In conclusion, we have developed a unified theory of nonequilibrium tension dynamics in stiff polymers based on the scale separation between the two dynamic correlation lengths $\ell_{\perp}$ and $\ell_{\|}$. The recovered known results and our new predictions are summarized in Figs. 2 and 3 and Table II. Various dynamic regimes should be well realizable for certain biopolymers and it is an intriguing question whether the tension propagation laws $\ell_{\|}(t)$ govern me- chanical signal transduction through the cytoskeleton $[6,20]$. Inclusion of hydrodynamic interactions merely produces logarithmic corrections but would give rise to more interesting effects for membranes. Other natural generalizations including the transverse nonlinear response and more complex force protocols (e.g., [17]) are currently also under investigation.

[1] T. T. Perkins, D. E. Smith, and S. Chu, Science 276, 2016 (1997); B. Maier, U. Seifert, and J. O. Rädler, Europhys. Lett. 60, 622 (2002); N.-K. Lee and D. Thirumalai, Biophys. J. 86, 2641 (2004).

[2] Y. Bohbot-Raviv, W.Z. Zhao, M. Feingold, C. H. Wiggins, and R. Granek, Phys. Rev. Lett. 92, 098101 (2004).

[3] U. Seifert, W. Wintz, and P. Nelson, Phys. Rev. Lett. 77, 5389 (1996).

[4] R. Everaers et al., Phys. Rev. Lett. 82, 3717 (1999).

[5] F. Brochard-Wyart, A. Buguin, and P.G. de Gennes, Europhys. Lett. 47, 171 (1999).

[6] V. Shankar, M. Pasquali, and D. C. Morse, J. Rheol. (N.Y.) 46, 1111 (2002).

[7] R. E. Goldstein and S. A. Langer, Phys. Rev. Lett. 75, 1094 (1995).

[8] F. MacKintosh, J. Käs, and P. Janmey, Phys. Rev. Lett. 75, 4425 (1995).

[9] R. Granek, J. Phys. II (France) 7, 1761 (1997); F. Gittes and F. C. MacKintosh, Phys. Rev. E 58, R1241 (1998).

[10] D. Morse, Macromolecules 31, 7044 (1998).

[11] $t_{\star}=\left(\zeta L^{2} / \ell_{p}\right)^{4}$ if $\mathfrak{\uparrow} \ll \ell_{p}^{2} / L^{4}$ and $t_{\star}=\left(\zeta L^{2} / \ell_{p}\right)^{2} \mathfrak{f}^{-1}$ if $\mathfrak{f} \gg \ell_{p}^{2} / L^{4}$; see Fig. 3 .

[12] Identifying $\ell_{\perp, \|}$ as correlation length of $\partial_{t} r_{\perp, \|}$, respectively, justifies the notation.

[13] O. Hallatschek, E. Frey, and K. Kroy, Phys. Rev. E 70, 031802 (2004).

[14] O. Hallatschek et al. (to be published).

[15] The right-hand side of this equation has recently independently been derived in a related context [2].

[16] Our identification of arclength averages with (local) ensemble averages after Eq. (3) breaks down for $\mathfrak{f}<$ $\left(\zeta / \ell_{p}\right)^{1 / 4} t^{-7 / 16}$, where fluctuations in the tension become comparable to its average [14].

[17] We postpone "mixed" scenarios (e.g., strong stretching of a prestretched polymer) that involve more than one external force scale. Contrary to previous expectations [3,5], they lead to multiple crossovers even for $t \gg t_{\mathfrak{f}}$.

[18] L. Le Goff et al., Phys. Rev. Lett. 89, 258101 (2002).

[19] C. Bustamante et al., Science 265, 1599 (1994).

[20] M. L. Gardel et al., Phys. Rev. Lett. 91, 158302 (2003). 